\title{
Video Streaming Using Whitespace Spectrum for Vehicular Applications
}

\author{
Tan Zhang, Sayandeep Sen, and Suman Banerjee \\ Department of Computer Sciences \\ University of Wisconsin-Madison, USA \\ \{tzhang, sdsen, suman\}@cs.wisc.edu
}

\begin{abstract}
We present Scout, a communication system leveraging TV whitespaces to support robust and high-speed streaming services. Scout uses two key techniques to improve video performance. First, it extends network coverage through an asymmetric network architecture where whitespace transceivers are used for the downlink direction while a cellular path is used for the uplink. Scout further leverages some unique opportunities that arise in vehicular systems. In particular, it sends a front radio to lookahead and identify the best channel parameters when the rear radio eventually reaches the forward post. We demonstrate the performance of Scout by using a single base station to stream a high-quality video to a vehicle driving along a $1.3 \mathrm{~km}$ road stretch.
\end{abstract}

\section{Categories and Subject Descriptors}

C.2.1 [Computer-Communication Networks]: Network Architecture and Design-Wireless communication

\section{Keywords}

TV Whitespaces, Vehicular Network, Video Streaming

\section{DESCRIPTION}

Video streaming is a vital on-board service that enables diverse road safety and infotainment applications for passengers. In this work, we explore using the emerging TV whitespaces spectrum for this purpose. This is not only because of the abundant spectral resource available for feeding the bandwidth-hungry video applications, but also for its excellent propagation characteristics especially suited for streaming to moving vehicles. To efficiently utilize TV whitespaces, we have recently developed a system called Scout [2] that consists of two key ideas.

(i) An asymmetric design for coverage improvement: Scout communicates the downlink traffic (usually the dominant fraction) primarily over the whitespace path while sending the uplink traffic primarily over existing cellular path as shown in Figure 1(a). The motivation of this design comes from the asymmetric transmission power limit in TV whitespaces. That is, the mobile client is allowed to transmit at much lower power-100 $\mathrm{mW}$ compared to the static base station allowed to- $4 W$. The ubiquitous cellular connectivity allows Scout to circumvent the "weak" whitespaces uplink, in turn extending the coverage of each base station to a maximum. (ii) A scouting radio for channel estimation: The operation of the asymmetric network is plagued by the high feedback latency in the

Copyright is held by the author/owner(s). MobiSys'13, June 25-28, 2013, Taipei, Taiwan. ACM 978-1-4503-1672-9/13/06.

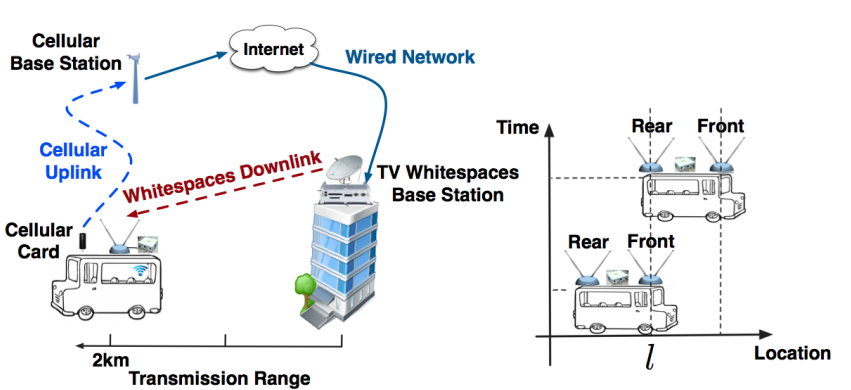

(a)

(b)

Figure 1: Scout design: (a) asymmetric architecture; (b) illustration of the "scouting" radio.

cellular uplink. The delayed feedback leads to poor transmission decisions by the base station. To tackle this problem, Scout uses a scouting radio mounted at the head of the vehicle (Figure 1(b)) to "scout" the likely channel condition at the future reception location of the rear radio. By the time the rear radio reaches the same location $l$, the base station can use the channel observation previously made by the front radio at $l$ to choose better transmission parameters for the rear radio. Built upon this estimation framework, we use multiple transmission techniques, i.e., rate adaptation, inter-packet FEC and traffic duplication, to enhance video delivery.

Video streaming using Scout: We showcase the video performance of Scout in [1] by streaming a high-quality (1080p) video from YouTube to a vehicle driving along a $1.3 \mathrm{~km}$ road stretch. The setup consists of one base station and one mobile client. The base station is mounted atop a 8-floor building. It is equipped with an Ethernet connection for fetching video traffic from Internet and relaying it over the asymmetric network. It achieves extensive downlink coverage of several kilometers by using a whitespaces radio, a high-gain power amplifier, and directional antennas. As the mobile client, we mount two whitespaces radios on the vehicle for receiving downlink traffic, while using a $3 \mathrm{G}$ cellular card for uplink communications. The video playback over Scout is interruption-free.

\section{Acknowledgments}

The authors were supported in part by the US National Science Foundation via awards CNS1040648, CNS0916955, CNS0855201, CNS0747177, CNS1064944, and CNS1059306.

\section{REFERENCES}

[1] Scout demo - an asymmetric vehicular network design over tv whitespaces. wings-www.cs.wisc.edu/projects/scout/.

[2] T. Zhang, S. Sen, and S. Banerjee. An asymmetric vehicular network design over tv whitespaces. In ACM HotMobile, 2013. 